\title{
Local allergic conjunctivitis: a phenotype of allergic conjunctivitis
}

\author{
Yasuo Yamana $(\mathbb{D})$ Ken Fukuda $\cdot$ Ryota Ko $\cdot$ Eiichi Uchio
}

Received: 19 November 2018/Accepted: 1 April 2019/Published online: 15 May 2019

(C) The Author(s) 2019

\begin{abstract}
Purpose Local allergic rhinitis (LAR) has been reported in the field of otolaryngology; however, the concept of local allergic conjunctivitis (LAC) has not been widely studied in the ophthalmologic community. We routinely examined total $\mathrm{IgE}$ levels in tear fluids ( $t$-tIgE) and antigen-specific IgE levels in serum $(s$-sIgE) in patients with suspected allergic conjunctivitis, on the basis of Japanese guidelines for allergic conjunctival diseases. There are several cases in which the results of $t$-tIgE and $s$-sIgE testing are divergent. We suggest that these divergent cases correspond to LAR in otolaryngology.

Methods The study included 148 patients (33 men and 115 women) with clinical symptoms and signs of allergic conjunctivitis. Allerwatch Tear $\operatorname{IgE}^{\circledR}$ was used for measurement of $t$-tIgE levels. ImmunoCAP Rapid $^{\circledR}$ and View Allergy $39^{\circledR}$ were used for measurement of $s$-sIgE levels. Conjunctival cytology
\end{abstract}

Y. Yamana $(\bowtie)$

Yamana Eye Clinic, 13-5, Nabeyama, Nakama,

Fukuoka 809-0022, Japan

e-mail: yamanaganka@yamana.or.jp

K. Fukuda

Department of Ophthalmology and Visual Science, Kochi

Medical School, Kochi, Japan

R. Ko $\cdot$ E. Uchio

Department of Ophthalmology, Faculty of Medicine,

Fukuoka University, Fukuoka, Japan using spatula was used to identify eosinophils on the conjunctiva.

Results A total of 83 patients $(56.1 \%)$ were positive and 65 patients were negative for $t$-tIgE in the AW. In the ICR, 97 patients $(65.5 \%)$ were positive for at least one of the eight allergens, whereas 51 (34.5\%) were negative for all allergens. Among 83 patients positive for $t$-tIgE, $14(16.9 \%)$ had no detectable $s$-sIgE. Therefore, we considered the possibility of LAC in cases in which only local IgE could be detected. Among 28 cases (18.9\%) who were negative for $t$-tIgE and $s$-sIgE, 21 underwent conjunctival scraping; eosinophils were found in four cases and eosinophilic granules in two. Accordingly, we considered the possibility of non-IgE-type AC in these six cases.

Conclusions These results suggest the existence of LAC that is a candidate of a phenotype of AC.

Keywords Allergic conjunctivitis - Phenotype · Serum total IgE $\cdot$ Specific serum IgE - Total tear IgE

\section{Introduction}

According to the Guidelines for the Clinical Management of Allergic Conjunctival Disease (ACD) of the Japanese Ophthalmology Society ("the guidelines") [1], allergic conjunctivitis (AC) is defined as nonproliferative conjunctivitis associated with a type I 
allergy mediated by IgE antibodies. The guidelines indicate that a definitive diagnosis of ACD requires positive results for serum antigen-specific $\operatorname{IgE}(s$-sIgE) antibodies and eosinophils in a conjunctival smear. A commercial kit that measures total IgE antibody levels in lacrimal fluid has recently become available; it can be used to confirm local production of $\operatorname{IgE}$ antibodies in the eye, but this test cannot be used for the definitive diagnosis of ACD.

Allergic diseases have various phenotypes [2]. In 1975, Huggins et al. [3] reported that allergic rhinitis (AR) has one entity, i.e., local allergic rhinitis (LAR). LAR is described as a phenotype of AR in the Global Atlas of Allergic Rhinitis and Chronic Rhinosinusitis of the European Academy of Allergy and Clinical Immunology [4]. In 1993, Leonardi et al. [5] suggested a probable entity of local allergic conjunctivitis (LAC), but the concept of LAC has not been widely studied in the ophthalmologic community.

We performed total tear $\operatorname{IgE}(t$-tIgE) and specific serum $\operatorname{IgE}(s$-sIgE) testing in patients clinically diagnosed using subjective symptoms (itchy, red, or watery eye) and clinical findings based on the guideline [1], and evaluated the results for the purpose of seeking the presence of LAC cases in these patients.

Moreover, we also evaluated cytological analysis of eosinophils in the conjunctival scraping specimens in patients who showed negative for both $t$-tIgE and $s$ sIgE to make clear the pathophysiology of LAC in this study.

\section{Materials and methods}

This study followed the ethical principles of the Declaration of Helsinki and was approved by the Ethics Committee of Clinical Research Network Fukuoka (No. 17-E01). The study was registered in the UMIN Clinical Trials Registry (UMIN Trial ID: UMIN000027651).

This study included 148 patients (33 men and 115 women; 5-88 years old; mean age, 65.3 years) who visited the Yamana Eye Clinic between December 2013 and March 2017, with a clinical diagnosis of AC on the basis of subjective and clinical symptoms (Table 1). Patients without $t$-tIgE and $s$-sIgE test results were excluded.

The following tests were performed according to the guidelines [1]. T-tIgE levels were measured with
Table 1 Age groups of the subjects, $n=148$

\begin{tabular}{lc}
\hline Age group (years) & $N(\%)$ \\
\hline$<10$ & $2(1.4)$ \\
$10-19$ & $1(0.7)$ \\
$20-29$ & $4(2.7)$ \\
$30-39$ & $9(6.1)$ \\
$40-49$ & $7(4.7)$ \\
$50-59$ & $15(10.1)$ \\
$60-69$ & $32(21.6)$ \\
$70-79$ & $57(38.5)$ \\
$\geq 80$ & $21(14.2)$ \\
\hline
\end{tabular}

the Allerwatch ${ }^{\circledR}$ Tear IgE (Wakamoto Pharmaceutical Co., Ltd., Tokyo, Japan) (AW) kit, using immunochromatography [6, 7]. S-sIgE antibody levels against eight allergens, included Dermatophagoides pteronyssinus, cockroach, cat dander, dog dander, Japanese cedar, orchard grass, ragweed, and mugwort, were measured with the ImmunoCAP ${ }^{\circledR}$ Rapid (Thermo Fisher Diagnostics K.K., Tokyo, Japan) (ICR) kit, using a fluorescent enzyme immunoassay $[8,9]$. Antibody levels against 39 allergens (including the eight allergens measured with ICR) were assessed with the View Allergy $39^{\circledR}$ (Thermo Fisher Diagnostics K.K., Tokyo, Japan) (View39) kit, using a chemiluminescent enzyme immunoassay (Table 2). The ICR uses immunochromatography based on a sandwich method, with colloidal gold as the labeled substance; the View39 also uses immunochromatography based on a sandwich method, but with $\beta$ galactosidase as the labeled substance.

All patients underwent both $t$-tIgE and $s$-sIgE testing. Of the patients who tested positive for $t$-tIgE but had no detectable $s$-sIgE antibodies, more than half underwent serum total IgE ( $s$-tIgE) testing.

Conjunctival cytology using spatula was carried out on the palpebral conjunctiva of patients with negative results in all tests; the specimens were examined for the presence of eosinophils using Hansel staining and an optical microscope (Olympus Corporation, Tokyo, Japan). 
Table 2 Allergens included in the specific IgE View Allergy 39 test kit

\begin{tabular}{llll}
\hline Inhaled and other allergens & Food allergens & \\
\hline House dust & Dermatophagoides pteronyssinus, house dust & Egg & Egg, ovomucoid \\
Pets & Cat dander, dog dander & Milk & Milk \\
Insects & Moth, cockroach & Wheat & Wheat \\
Trees & Japanese cedar, Japanese cypress, Alder, Black birch & Beans/grains/seeds & $\begin{array}{c}\text { Peanuts, soybeans, } \\
\text { buckwheat, sesame, rice }\end{array}$ \\
Herbs/grasses & Orchard grass, ragweed, mugwort, Timothy & Crustaceans & Shrimp/lobster, crab \\
Airborne fungi & Alternaria, Aspergillus & Fruit & Kiwis, apples, bananas \\
Fungi and others & Candida, Malassezia, latex & Fish/meat & Tuna, salmon, mackerel, \\
& & & beef, chicken, pork \\
\hline
\end{tabular}

The text in bold reflects food allergens with mandatory or recommended labeling in Japan

\section{Results}

A total of 83 patients (56.1\%) tested positive for $t$-tIgE in the AW. In the ICR, 97 patients (65.5\%) were positive for at least one of the eight allergens, whereas $51(34.5 \%)$ were negative for all allergens. Of the 83 patients who tested positive for $t$-tIgE in the AW, 19 $(22.9 \%)$ were negative for all eight allergens examined with the ICR. Of these, five had detectable $s$-sIgE antibodies in View39, whereas 14 (16.9\%) had no detectable $s$-sIgE antibodies (Table 3). Of these 14 patients, nine underwent $s$-tIgE testing; all showed a level that was below the reference range $(<170 \mathrm{IU} /$ $\mathrm{mL}$; Table 4).

Of 32 patients $(21.6 \%)$ with negative results in both the AW and the ICR, four had detectable $s$-sIgE antibodies in View39 against allergens that were not examined in the ICR. In total, 28 patients $(18.9 \%)$ were negative in all three tests (AW, ICR, and View39).

Of these 28 patients who were negative for $t$-tIgE and $s$-sIgE antibodies, 21 underwent conjunctival cytology of the palpebral conjunctiva to evaluate the presence of eosinophils. Eosinophils and eosinophilic granules were detected in four (19\%) and two $(9.5 \%)$
Table 4 Clinical details of patients who were positive for total tear $\operatorname{IgE}$ and showed no detectable specific serum $\operatorname{IgE}$ antibodies

\begin{tabular}{llllll}
\hline No. & Age (years) & Sex & $t$-tIgE & $s$-sIgE & $s$-tIgE \\
\hline 1 & 76 & $\mathrm{~F}$ & + & - & Not tested \\
2 & 80 & $\mathrm{~F}$ & + & - & $75 \mathrm{IU} / \mathrm{mL}$ \\
3 & 74 & $\mathrm{~F}$ & + & - & $31 \mathrm{IU} / \mathrm{mL}$ \\
4 & 84 & $\mathrm{~F}$ & + & - & Not tested \\
5 & 51 & $\mathrm{~F}$ & + & - & Not tested \\
6 & 79 & $\mathrm{~F}$ & + & - & $7 \mathrm{IU} / \mathrm{mL}$ \\
7 & 77 & $\mathrm{~F}$ & + & - & $37 \mathrm{IU} / \mathrm{mL}$ \\
8 & 75 & $\mathrm{~F}$ & + & - & $9 \mathrm{IU} / \mathrm{mL}$ \\
9 & 74 & $\mathrm{~F}$ & + & - & $3 \mathrm{IU} / \mathrm{mL}$ \\
10 & 55 & $\mathrm{M}$ & + & - & Not tested \\
11 & 85 & $\mathrm{~F}$ & + & - & Not tested \\
12 & 71 & $\mathrm{~F}$ & + & - & $11 \mathrm{IU} / \mathrm{mL}$ \\
13 & 69 & $\mathrm{~F}$ & + & - & $48 \mathrm{IU} / \mathrm{mL}$ \\
14 & 80 & $\mathrm{~F}$ & + & - & $2 \mathrm{IU} / \mathrm{mL}$ \\
\hline
\end{tabular}

$s$-sIgE, Specific serum IgE; $s$-IIgE, Serum total IgE; $t$-tIgE, Total tear $\operatorname{IgE}$

patients, respectively. Of the 28 patients, 19 underwent $s$-tIgE testing; of these, 17 had a normal value $(<170 \mathrm{IU} / \mathrm{mL})$ and two had a level $\geq 170 \mathrm{IU} / \mathrm{mL}$.

Table 3 The results of total tear IgE and specific serum IgE test

\begin{tabular}{lllr}
\hline & $t$-tIgE Positive & $t$-tIgE Negative & Total \\
\hline$s$-sIgE Positive & 69 cases $(83.1 \%)$ & 37 cases $(56.9 \%)$ & 106 cases $(71.6 \%)$ \\
$s$-sIgE Negative & 14 cases $(16.9 \%)$ & 28 cases $(43.1 \%)$ & 42 cases $(23.4 \%)$ \\
Total & 83 cases $(100 \%)$ & 65 cases $(100 \%)$ & 148 cases $(100 \%)$ \\
\hline
\end{tabular}

$s$-sIgE, Specific serum IgE; $t$-tIgE, Total tear IgE 


\section{Discussion}

The guidelines define ACD as "conjunctival inflammatory diseases associated with type I allergy accompanied by some subjective and objective symptoms" [1]. The presence of clinical symptoms, type I allergic diathesis, and a type I allergic reaction in the conjunctiva are required for a diagnosis of AC. Type I allergic diathesis can be confirmed via positive results for serum antigen-specific IgE antibody levels or a skin reaction with presumed antigens, an increase in serum total IgE antibody levels, the presence or the absence of a family history of allergic diseases, and comorbidities of other allergic diseases. The guidelines state that the presence of eosinophils in the conjunctiva must be shown for the definitive diagnosis of ACD.

Leonardi et al. [5] stated: "In patients with rhino conjunctivitis or rhinitis, and in 10 normal subjects, results of conjunctival provocation tests (CPT) and nasal-specific provocation test (NPT) were in $100 \%$ agreement." Conversely, in patients with only conjunctivitis, little correlation was found between the results of CPT and NPT $(K=0.3)$. Tear-specific IgE was the only positive diagnostic sign of antigen sensitivity in $35 \%$ of VKC (vernal keratoconjunctivitis) patients and $30 \%$ of AC patients. It was suggested from this that the conjunctiva can be a uniquely sensitized target organ in allergic patients [5]. In the field of otolaryngology, the entity of LAR was first reported by Huggins et al. in 1975 [3] and subsequently by Rondón et al. in 2010 [10, 11] as well as by Campo et al. [12]. Rondón and colleagues [10, 11] reported that atopic AR was identified in 63\%, nonallergic AR in $11 \%$, and LAR in $26 \%$ of Spanish adult patients with rhinitis. LAR is characterized by symptoms of rhinitis (sneezing, watery rhinorrhea, and nasal congestion), eosinophilic infiltration of the nasal mucosa, and $\operatorname{IgE}$ antibodies localized in the nasal mucosa. However, LAR shows no elevation of serum IgE levels; it is likely to progress to atopic AR accompanied by asthma or other complications. After that Rondón and colleagues reported that a similar rate of development of AR with systemic atopy was detected in patients and controls $(9.7 \%$ vs $7.8 \%$, longrank $P=0.623)$. In five patients, conversion to systemic atopy occurred $>10$ years $(3 \%)$. And then Rondón et al. [13] concluded as follow: LAR is a welldifferentiated clinical entity with a low rate of development of systemic atopy, a natural evolution toward worsening and a risk factor for suffering asthma. Campo et al. [14] reviewed the diagnosis, classification and etiology, therapeutic options, and they concluded as follows: LAR rapidly evolves toward the clinical worsening and the association to asthma and conjunctivitis implying that an early diagnosis and the initiation of specific therapies are crucial for controlling the disease and potentially preventing its comorbidities.

Kato et al. [15] and Gelardi et al. [16] reported on the etiology of LAR. Kato investigated the pathophysiology of mice with allergic rhinitis that initially sensitized with ragweed pollen through the nasal route. He concluded that $\mathrm{Th} 2$ cells cause nasal symptoms due to the accumulation of Th2 cell in the nasal region on the pathology of LAR. Gelardi hypothesized that the nasal IgE production represents a form of spontaneous immune response.

In this study, among 83 patients positive for $t$-tIgE, $14(16.9 \%)$ had no detectable $s$-sIgE antibodies; however, not all $s$-sIgE antibodies could be examined. Of these 14 patients, nine underwent $s$-tIgE testing; all showed a level that was below the reference range. Therefore, the prevalence of LAC was $16.9 \%$ in this study. Atopic bronchial asthma [17-19], atopic dermatitis (AD) [20, 21], and AR [22] have various phenotypes. LAC might be a candidate of novel phenotype in AC.

Several ophthalmologic studies have reported local cytokine dynamics in the eye, including the palpebral conjunctiva and lacrimal fluid [23-28]. However, few studies have been reported for the dissociation of serum and tear IgE antibody levels in patients with AC. Aghayan-Ugurluoglu and colleagues [29] reported that serum and tears of many of the pollenallergic individuals with conjunctivitis exhibited specificity for the very same pollen allergens, but no allergen-specific IgE antibodies were detected in tears of non-atopic individuals. They also stated that $\operatorname{IgA}$ antibodies in sera and tears of patients with allergic conjunctivitis were mainly directed against non-allergenic moieties and showed specificities that were significantly different from those of IgE antibodies [29]. Whereas these results were interesting to consider the local allergic reactions in clinical situation of ACD, their report did not directly support our findings. Pathophysiological property of LAC has not been clarified, because the prevalence of LAC among 
$\mathrm{AC}$ is limited and several steps of diagnostic measures, serum, and tear antigen-specific IgE tests and conjunctival cytology test added to clinical evaluation, are required for the diagnosis of LAC. However, Leonardi et al. [30] reported that while normal subjects resulted negative for the presence of specific IgE both in serum and in tears, of the $10 \mathrm{VKC}$ patients, six resulted positive to specific IgE in serum and/or tears, and in three of these six patients, specific IgE was found positive only in tears. Also, study population was different from our study; it was revealed that the dissociation of serum and tear IgE might be observed in some patients among ACD. Future study will be focused on immunological analysis of ocular surface in LAC to find out its allergological specificities compared with other ACD.

We also found cases who showed positive eosinophils in the conjunctival scrapings, but who had negative $t$-tIgE and $s$-sIgE tests among several clinically diagnosed AC patients. There seems no study focusing on these specific cases that showed only positive conjunctival eosinophils without antigenspecific IgE elevation in both serum and tears. Although we considered the possibility of the presence of non-IgE-type AC in these cases, pathological bases of this phenotype will also be clarified in the future, since non-IgE-type AC is considered rarer in $\mathrm{AC}$ cases.

\section{Conclusion}

$\mathrm{AC}$ has been considered as an ocular manifestation of type I allergy mediated by IgE antibodies. However, the results of the present study suggest the existence of LAC, as a novel phenotype of AC. We also found nonIgE-type $\mathrm{AC}$ as another unique clinical entity of $\mathrm{AC}$ in this study.

Acknowledgements We sincerely thank Dr. Sadafumi Tamiya, Chief Manager of Department of Pathological Diagnosis, Kitakyushu City Medical Center, for his advice about the eosinophil exam and help in identifying eosinophils.

\section{Compliance with ethical standards}

Conflict of interest All authors declare that there is no conflict of interest regarding this publication of this paper.

Ethical approval All procedures performed in studies involving human participants were in accordance with the ethical standards of the institutional and/or national research committee and with the 1964 Declaration of Helsinki and its later amendments or comparable ethical standards.

Informed consent Informed consent was obtained from all individual participants included in the study.

Open Access This article is distributed under the terms of the Creative Commons Attribution 4.0 International License (http:// creativecommons.org/licenses/by/4.0/), which permits unrestricted use, distribution, and reproduction in any medium, provided you give appropriate credit to the original author(s) and the source, provide a link to the Creative Commons license, and indicate if changes were made.

\section{References}

1. Takamura E, Uchio E, Ebihara N, Ohno S, Ohashi Y, Okamoto S, Kumagai N, Satake Y, Shoji J, Nakagawa Y, Namba K, Fukagawa K, Fukushima A, Fujishima H, Japanese Society of Allergology (2017) Japanese guideline for allergic conjunctival diseases. Allergol Int 66(2):220-229

2. Novak N, Bieber T (2003) Allergic and nonallergic forms of atopic diseases. J Allergy Clin Immunol 112(2):252-262

3. Huggins KG, Brostoff J (1975) Local production of specific $\mathrm{IgE}$ antibodies in allergic-rhinitis patients with negative skin tests. Lancet 306(7926):148-150

4. Rondón C (2017) Local allergic rhinitis. Global atlas of allergic rhinitis and chronic rhinosinusitis: the EAACI, pp 237-240. http://www.eaaci.org/resources/3343-globalatlas-of-allergic-rhinitis-and-chronic-rhinosinusitis.html.

Last updated 28 April 2017. http://www.eaaci.org/ globalatlas/ENT_Atlas_web.pdf

5. Leonardi A, Battista MC, Gismondi M, Fregona IA, Secchi AG (1993) Antigen sensitivity evaluated by tear-specific and serum-specific IgE, skin tests, and conjunctival and nasal provocation tests in patients with ocular allergic disease. Eye 7:461-464

6. Inada N, Shoji J, Kato H, Kiely S, Mulyanto, Sawa M (2009) Clinical evaluation of total $\operatorname{IgE}$ in tears of patients with allergic conjunctivitis disease using a novel application of the immunochromatography method. Allergol Int 58(4):585-589

7. Mimura FT, Usui T, Mori M, Funatsu H, Noma H, Aixinjueluo W, Yamamoto H, Amano S (2010) Rapid immunochromatography of total tear immunoglobulin $\mathrm{E}$ in allergic conjunctivitis with Allerwatch. J Investig Allergol Clin Immunol 20(7):627-628

8. Eigenmann PA, Kuenzli M, D’Apuzzo V, Kehrt R, Joerg W, Reinhardt M, Rudengren M, Borres MP, Lauener RP (2009) The ImmunoCAP ${ }^{\circledR}$ Rapid Wheeze/Rhinitis Child test is useful in the initial allergy diagnosis of children with respiratory symptoms. Pediatr Allergy Immunol 20(8):772779

9. Komiyama K, Masumoto A, Nishihara F, Kobayashi T, Soma T, Hagiwara K, Kanazawa M, Nagata M (2013) Rapid specific IgE assay (ImmunoCAP ${ }^{\circledR}$ RAPID) and skin prick test in the diagnosis of allergic disease. Arerugi 62(12):1631-1641 
10. Rondón C, Fernandez J, Canto G, Blanca M (2010) Local allergic rhinitis: concept, clinical manifestations, and diagnostic approach. J Investig Allergol Clin Immunol 20(5):364-371

11. Rondón C, Campo P, Togias A, Fokkens WJ, Durham SR, Powe DG, Mullol J, Blanca M (2012) Local allergic rhinitis: concept, pathophysiology, and management. J Allergy Clin Immunol 129(6):1460-1467

12. Campo P, Rondón C, Gould HJ, Barrionuevo E, Gevaert P, Blanca M (2015) Local IgE in non-allergic rhinitis. Clin Exp Allergy 45(5):872-881

13. Rondón C, Campo P, Eguiluz-Gracia I, Plaza C, Bogas G, Galindo P, Mayorga C, Torres MJ (2018) Local allergic rhinitis is an independent rhinitis phenotype: the results of a 10-year follow-up study. Allergy 73(2):470-478

14. Campo P, Eguiluz-Gracia I, Bogas G, Salas M, Plaza Serón C, Pérez N, Mayorga C, Torres MJ, Shamji HM, Rondón C (2019) Local allergic rhinitis: implications for management. Clin Exp Allergy 49(1):6-16

15. Kato Y, Akasaki S, Muto-Haenuki Y, Fujieda S, Matsushita K, Yoshimoto T (2014) Nasal sensitization with ragweed pollen induces local-allergic-rhinitis-like symptoms in mice. PLoS ONE 9(8):e103540

16. Gelardi M, Guglielmi AV, Iannuzzi L, Quaranta VN, Quaranta N, Landi M, Correale M, Sonnante A, Rossini M, Mariggiò MA, Canonica GW, Passalacqua G (2016) Local allergic rhinitis: entopy or spontaneous response? World Allergy Organ J 9(1):39

17. Wenzel SE (2006) Asthma: defining of the persistent adult phenotypes. Lancet 368(9537):804-813

18. Moore WC, Meyers DA, Wenzel SE, Teague WG, Li H, Li X, D'Agostino R Jr, Castro M, Curran-Everett D, Fitzpatrick AM, Gaston B, Jarjour NN, Sorkness R, Calhoun WJ, Chung KF, Comhair SA, Dweik RA, Israel E, Peters SP, Busse WW, Erzurum SC, Bleecker ER, National Heart, Lung, and Blood Institute's Severe Asthma Research Program (2010) Identification of asthma phenotypes using cluster analysis in the Severe Asthma Research Program. Am J Respir Crit Care Med 181(4):315-323

19. Agache I, Akdis C, Jutel M, Virchow JC (2012) Untangling asthma phenotypes and endotypes. Allergy 67(7):835-846

20. Tokura Y (2010) Extrinsic and intrinsic types of atopic dermatitis. J Dermatol Sci 58(1):1-7

21. Katayama I, Aihara M, Ohya Y, Saeki H, Shimojo N, Shoji S, Taniguchi M, Yamada H, Japanese Society of Allergology (2017) Japanese guidelines for atopic dermatitis 2017. Allergol Int 66(2):230-247
22. Agache I (2017) Penotypes and endotypes of allergic rhinitis. Global atlas of allergic rhinitis and chronic rhinosinusitis: the EAACI, pp 268-270. http://www.eaaci.org/ resources/3343-global-atlas-of-allergic-rhinitis-andchronic-rhinosinusitis.html. Last updated 28 April 2017

23. Uchio E, Ono SY, Ikezawa Z, Ohno S (2000) Tear levels of interferon-gamma, interleukin (IL)-2, IL-4 and IL-5 in patients with vernal keratoconjunctivitis, atopic keratoconjunctivitis and allergic conjunctivitis. Clin Exp Allergy 30(1):103-109

24. Fukagami K, Nakajima T, Saito H, Tsubota K, Shimmura S, Natori M, Hirai K (2000) IL-4 induces eotaxin production in corneal keratocytes but not in epithelial cells. Int Arch Allergy Immunol 121(2):144-150

25. Kumagai N, Fukuda K, Ishimura Y, Nishida T (2000) Synergistic induction of eotaxin expression in human keratocytes by TNF- $\alpha$ and IL-4 or IL-13. Invest Ophthalmol Vis Sci 41(6): 1448-1453

26. Matsuda A, Okayama Y, Terai N, Yokoi N, Ebihara N, Tanioka H, Kawasaki S, Inatomi T, Katoh N, Ueda E, Hamuro J, Murakami A, Kinoshita S (2009) The role of interleukin-33 in chronic allergic conjunctivitis. Invest Ophthalmol Vis Sci 50(10):4646-4652

27. Fukuda K, Fujitsu Y, Seki K, Kumagai N, Nishida T (2003) Differential expression of thymus-and activation-regulated chemokine (CCL17) and macrophage-derived chemokine (CCL22) by human fibroblasts from cornea, skin, and lung. J Allergy Clin Immunol 111(3):520-526

28. Sugita J, Asada Y, Ishida W, Iwamoto S, Sudo K, Suto H, Matsunaga T, Fukuda K, Fukushima A, Yokoi N, Ohno T, Azuma M, Ebihara N, Saito H, Kubo M, Nakae S, Matsuda A (2017) Contributions of Interleukin-33 and TSLP in a papain-soaked contact lens-induced mouse conjunctival inflammation model. Immun Inflamm Dis 5(4):515-525

29. Aghayan-Ugurluoglu R, Ball T, Vrtala S, Schweiger C, Kraft D, Valenta R (2000) Dissociation of allergen-specific $\mathrm{IgE}$ and $\mathrm{IgA}$ responses in sera and tears of pollen-allergic patients: a study performed with purified recombinant pollen allergens. J Allergy Clin Immunol 105(4):803-813

30. Leonardi A, Borghesan F, Faggian D, Plebani M (2015) Microarray-based $\operatorname{IgE}$ detection in tears of patients with vernal keratoconjunctivitis. Pediatr Allergy Immunol 26(7):641-645

Publisher's Note Springer Nature remains neutral with regard to jurisdictional claims in published maps and institutional affiliations. 